\title{
431 - DEMENTIA IN PRIMARY CARE AND DOCTOR-PATIENT-CARER INTERACTIONS: PRELIMINARY FINDINGS
}

\author{
Conceição Balsinha \\ (first author) \\ Comprehensive Health Research Centre (CHRC) \\ Nova Medical School, Universidade Nova de Lisboa, Lisboa, Portugal \\ Steve lliffe \\ University College London, UK \\ Sónia Dias \\ Comprehensive Health Research Centre (CHRC) \\ National School of Public Health, Universidade Nova de Lisboa, Lisboa, Portugal

\section{Manuel Gonçalves-Pereira} \\ (corresponding author: gpereira@nms.unl.pt) \\ Comprehensive Health Research Centre (CHRC) \\ Nova Medical School, Universidade Nova de Lisboa, Lisboa, Portugal
}

INTRODUCTION: Primary care visits of persons with dementia involve different types of communication, bringing together the patient, the family carer and the general practitioner (GP). A particular challenge is the necessary involvement of a third person (the carer) in patient-doctor encounters (or the patient in carer-doctor encounters, as dementia advances). These triad dynamics should be better understood, as health outcomes are expected to result from or be mediated by them.

OBJECTIVE: Our aim is to explore triadic dynamics in Portuguese primary care consultations with persons with dementia, their family carers and GPs.

METHODS: This is the first part of an ongoing project (Dementia in Primary Care: the Patient, the Carer and the Doctor in the Medical Encounter - Bayer Investigation Grant | NOVAsaúde Ageing 2018). Consultations with persons with dementia, their carers and GPs (purposive sampling) are audiorecorded, transcribed verbatim and thematically analysed. We report the analysis of interactions of the first six consultations, using NVIVO ${ }^{\circledR}$ software.

RESULTS: The most frequent type of interaction was between GPs and carers, followed by interactions involving the whole triad. The patients who had more recent relationships with their GPs tended to participate less, irrespective of the stage of dementia. Carers were the ones most often initiating triadic interactions, and GPs the ones most often terminating them by directly addressing the patients. Doctorcarers interactions were very sparse in some consultations.

DISCUSSION AND CONCLUSION: These preliminary findings suggest that doctor-patient interactions may be limited in a number of GPs' consultations, seemingly compromising patient-centred approaches. Nevertheless, even when GPs were involved in triadic interactions they often tried to address the patient directly. We are looking forward to complete this part of the project: to our knowledge, there is practically no evidence from live-recorded primary care consultations about these triadic dynamics. 